\title{
Teaching Psychology to Trainee Psychiatrists
}

\author{
John Hall, Principal Clinical Psychologist, The Warneford Hospital, Oxford
}

Psychological knowledge is important to psychiatrists, both because of the psychological concepts central to some fields, such as normal human development, and because of the psychological procedures widely used in both the assessment and treatment of patients. Psychology is taught to trainee psychiatrists as part of their preparation for the MRCPsych examination, mainly through the teaching for the Preliminary Test of the examination which covers the 'sciences basic to psychiatry'. In practice, the content of the test covers basic psychopathology and the neurosciences, as well as the behavioural and social sciences. While the Royal College of Psychiatrists does not itself produce a detailed syllabus for the examination, the Association of University Teachers of Psychiatry (AUTP) has prepared guidelines for students and teachers for these subjects. Since the Royal College has 'commended' these guidelines and published them (Bulletin, April 1982, 6, 54-56) they have a semiofficial status.

As concern continues about how best to teach the behavioural sciences to trainee psychiatrists, the Joint Standing Committee of the Royal College of Psychiatrists and the British Psychological Society agreed to carry out a survey of current practice in teaching psychology to trainee psychologists. This report presents the findings of that survey, some recommendations arising from it, and some qualifications of those recommendations in the light of the recent Royal College proposals for review of the structure of the MRCPsych. ${ }^{1}$

The survey was carried out in two stages and was routed via Professors of Psychiatry. The first stage, carried out in late 1983, focused on a description of current practice. Responses were obtained from 15 centres, and these responses were discussed by the Joint Standing Committee early in 1984, and led to a more structured follow-up survey late in 1984. This second stage provided 13 responses, and subsequent comments on an interim version of this report mean that in total 20 centres have contributed to the survey (Birmingham, Leeds, Leicester, Liverpool, Manchester, Nottingham, Oxford, Sheffield, Southampton: seven London centres (Barts, Guy's Institute of Psychiatry, The London, Royal Free, St Mary's, and University College); Edinburgh, Dundee; Belfast; Cardifi).

The survey yielded factual data about the teaching of psychology and behavioural sciences to trainee psychiatrists, and elicited a wide range of comments about the assumptions commonly made by both teachers and taught, and about the objectives and philosophy of such teaching. These comments provided a context within which to interpret the more factual data.

The AUTP guidelines recognise some of the difficulties of reconciling the knowledge that can be assumed from a recent graduate of a British Medical School, and the know- ledge possessed by some older trainees from abroad. The guidelines also recognise the balance required between a broad knowledge of the general field, and a detailed knowledge of some central topics or areas of recent research development. These difficulties are not unique to the education of trainee psychiatrists, but are certainly felt keenly by them. ${ }^{2}$ The clear distinction between psychological topics and, for example, sociological topics suggested by the AUTP guidelines does not necessarily match up with the way some courses are taught. A 'behavioural sciences' model is explicit in a few courses and implicit in several, so that, for example, ethology may be taught alongside child development, and social psychology may embrace some topics, such as illness behaviour, designated as sociology.

\section{Current practice in teaching}

The 15 responses from the first stage of the survey provided a clear picture of the way in which teaching is currently conducted. Most Preliminary Test (PT) courses (13) were designed explicitly for the examination, and consisted of 50-60 minute slots within a once-a-week day or afternoon of teaching. All but one of the courses distributed teaching over three notional 'terms', the one other centre arranging all teaching within a five month period and offering the same course twice a year. Courses ranged from $\mathbf{5 0}$ to 102 hours in total, although the longest course included 27 hours of 'Neurosciences' which were not included in the other MRCPsych PT courses. Within this overall total, psychological topics accounted for between $25 \%$ and $50 \%$ of the whole, with a mean of $39 \%$. Fourteen courses indicated the time devoted specifically to psychological topics: for the 13 courses providing developed coverage of different topics, six took between 14 and 20 hours, one took 24 hours, and the remaining six took between 28 and 29 hours, with a mean course time of 25 hours.

It was possible to carry out a detailed content analysis of 14 courses: psychologists taught all the statistics or experimental design content on a few courses, but that content is not included in this analysis. Content was classified into six main categories.

(1) Human development: topics on human development took up to one third of all the psychological teaching time, with a maximum time allocation of 11 hours. All but one course covered material from childhood to old age, the exception covering child development alone.

(2) Cognitive processes: this umbrella title covered several topics such as memory and perception that were given in 12 courses, and in a few courses was associated with teaching on psychiatric aspects of those processes. Maximum time allocated was seven hours.

(3) Individual differences and assessment: teaching on intelligence and personality was usually distinguishable 
from other cognitive processes, and was often combined with teaching on assessment methodology, or practice. All courses offered some teaching on these topics, up to a maximum of five hours for assessment, considered alone, and four hours for intelligence and personality considered together.

(4) Learning theory and behaviour therapy: three courses offered a package with exactly this title, and another seven courses offered teaching on these topics, the remainder indicating that specific therapeutic techiques were covered in the part II course or in associated clinical seminars. Up to nine hours were offered on these topics, considered together.

(5) Ethology: eight courses offered up to three hours on ethology.

(6) Social psychology: eight courses offered up to two hours on social psychological topics.

(7) Other topics: a range of other topics were included by more than one course, such as psychophysiology or the psychology of mental handicap. A few topics were taught by only one course, such as hypnosis.

A number of details of course organisation were given. Five courses provided an introductory section of up to four hours, content unspecified, to the psychological teaching. The topics covered were often indicated as being expanded as seminars in the second and third year of the Membership course. Several centres made oblique or direct remarks indicating difficulties they experienced in finding suitable teachers.

\section{Recommendations of changes in teaching}

The initial survey generated a number of provisional recommendations about the content and organisation of teaching psychology to trainee psychiatrists; these recommendations led to the questions of the second stage survey, which produced 33 responses. Table I indicates the suggested content distribution for a 20-hour psychology course, or for a 40-hour psychology course. Overall, this suggests that central psychological processes ( $21 \%$ ), learning, with clinical applications (18\%), and human development (17\%) might constitute about $60 \%$ of a course, with assessment concepts and methods (16\%), social psychology $(8 \%)$ and other topics making up the remaining $40 \%$. This percentage analysis of content might be useful in assigning questions within the 'about a third' of total content allocation to psychology implied by the AUTP guidelines.

Detailed comments on content were also solicited, and the most heavily supported recommendations or those which suggest a modification of the AUTP guidelines are highlighted, providing more detail than the Royal College proposals.

Some of the content suggestions imply only changes of detail in the outline provided in the AUTP guidelines. The section on human development should also ensure coverage of cognitive and language development, and cover some topics in adolescence and middle life. The section on psychology of learning should include the concept and technique of behavioural analysis. The study of memory should emphasise common clinical disorders of memory and their psychological interpretation. Recommendations about coverage of topics on assessment suggest a distinction between information on assessment practice, and psychometric theory. Familiarity with some of the most important and common assessment instruments is required, including some neuropsychological assessment techniques. Aspects of psychometric theory, such as concepts and calculations of reliability and validity, are taught by some courses in the statistics section and do not need to be repeated.

More major recommendations were made regarding topics in social psychology, and in the field of behavioural medicine. The recommendations regarding social psychology suggest the importance of interpersonal perception and social interaction as a foundation for teaching on social skills, and the importance of attribution theory and health beliefs. There was very strong support for a number of specific topics, such as stress, arousal, and biofeedback

TABLE I

Suggested allocation of psychological content in training courses (mean \% of time)

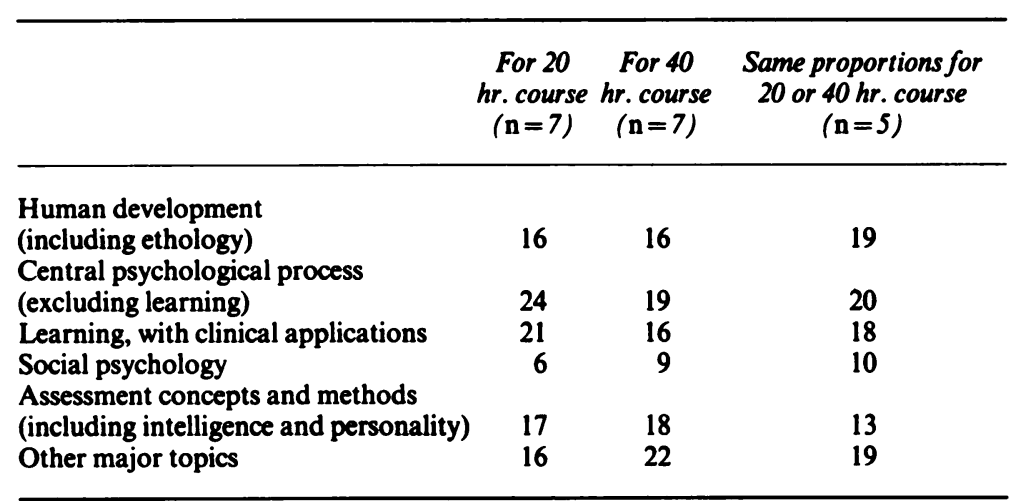


which perhaps have been viewed as psychophysiological, but which might now be considered as topics within 'behavioural medicine', as further illustrated by suggestions for teaching applied topics such as pain management and psychological aspects of sleep.

There was no clear statement about the need for an introduction to psychology. Three respondents agreed that an introductory course might be needed for some weaker or older candidates, but that most recent UK graduates had an adequate background knowledge in psychology. Every respondent in the second stage asked that more information be available about the MCQ questions, suggesting that guidance be given on the level of detail required, and that up-to-date examples are provided. Three respondents were strongly critical of the MCQ format, saying, for example, that questions ranged from 'definitely noddy, to the irrelevant and esoteric'. The reading lists supplied by the College are very detailed and do suggest basic textbooks for some topics. The survey asked for recommended textbooks: Hilgard and Atkinson's text was clearly the most popular, mentioned by 11 respondents in both the first and second stage surveys. Four respondents mentioned Weinman's Outline of Psychology as Applied Medicine, and two other titles were mentioned by two respondents (Psychology and Medicine, Griffiths, and Psychology and Medical Care, Kent and Dalgeish). If indeed most teachers believe trainees have an adequate knowledge of basic psychology, it is interesting that an American undergraduate text is so popular, and suggests that 'purpose-built' texts, as suggested by two respondents, might be worth considering.

Most teachers favour seminar-type presentations of material, especially for more able trainees, but conceded that some lecturing was inescapable. A number of respondents emphasised the strong pressure exerted by trainees themselves to give lectures, emphasising broad general knowledge. The value of well-prepared videos was mentioned. There was a preference for psychological topics to be distributed over the whole three years of formal teaching. rather than in one year, and to be integrated with associated psychiatric topics, which would be achieved by the new Royal College proposals. Only four respondents had any difficulty in obtaining teachers for particular topics, two mentioning social psychology teaching, but difficulty in obtaining teachers for other topics, such as ethology, behavioural genetics, and psychiatric anthropology must surely be encountered. Formal teaching for the PT is often supplemented by seminars, journal clubs, and supervised instruction in psychological techniques, but these arrangements are highly variable. Clearly the opportunity at least to observe psychological procedures in action can only reinforce the classroom learning which has taken place. Most clinical psychologists would probably encourage the direct training in psychological techniques of psychiatrists, although for some this raises major issues of principle. ${ }^{3}$

\section{Conclusions}

(1) The grounding in general psychology possessed by recent British medical graduates is usually considered adequate, but consideration could be given to offering an optional or refresher introduction to psychology, which need not exceed two hours in length.

(2) Variation in teaching method within a course is important, both to maintain trainee interest, and to achieve the dual objectives of achieving general field coverage and knowledge of some techniques or concepts in details. Seminar presentations are best arranged after field coverage of the topic area.

(3) The surveys together suggest a broad categorisation of content of a psychology course:

$\begin{array}{lr}\text { Central psychological processes } & 20 \% \\ \text { Learning and clinical applications } & 20 \% \\ \text { Human development } & 20 \% \\ \text { Assessment concepts and methods } & 15 \% \\ \text { Other topics (about one third of which would be } \\ \text { social psychology) } & 25 \%\end{array}$

These topics would be covered within the 'about one third' of the total PT course, but the AUTP guidelines do not indicate overall content allocation within a course, the amount of time given to psychopathology being especially unclear. More detailed content changes are recommended in the body of this report.

(4) The MCQ examination in particular attracted a large number of comments. Those comments have the following implications:

(a) further guidance on the allocation of content in the examination should be given. The new Royal College proposals do not give any guidance on the balance of content allocation in the first MCQ paper for the Membership Examination between the eight 'Sciences basic to psychiatry'. Presumably both teachers and trainees would benefit from some guidance in this direction, both for better organisation of teaching and better examination preparation;

(b) further guidance on the level of detail expected in answers should be given;

(c) up-to-date examples should be available, recognising the effort required to generate suitable MCQ questions.

(5) Distribution of this content over the two years of the new Membership examination will allow greater integration between the psychological concepts, details of psychological practice, and related psychiatric topics. In the intervening years before the new proposals are introduced, there is scope for more integration of psychological topics and associated psychiatric topics in the arrangement of teaching. While the new proposals will facilitate such integration, it should be noted that the 'basic sciences' content will be examined separately from clinical topics.

(6) Consideration could be given to constructing a list of recommended texts while not recommending any one 'set text'. The detailed content of courses suggests different orientations are taken towards some core areas of content, so recommended texts could ensure consistency in at least parts of a course while not hiding controversy.

(7) Trainee psychiatrists should be given the opportunity 
to observe psychological procedures in practice, and preferably to participate with supervision in psychological treatments.

Constraints of different placements and attachments may make it difficult to provide such practical experience at the same time as the related academic teaching.
ReFERENCES

'Royal College of Psychiatrists (1985) Working party for review of the MRCPsych: Report to the Court of Electors.

${ }^{2}$ Kopeluan, M. D. (1982) The psychiatrist learning psychology: sponge or jackdaw? AUTP Newsletter, December, 44-51.

${ }^{3}$ Fraser, D. (1984) The province of behaviour therapy. DCP Newsletter No. 46, 26-28.

\section{Drug Abuse in the Bahamas}

Nelson Clarke, Consultant Psychiatrist, and Michael Neville, Consultant Psychiatrist, Sandilands Rehabilitation Centre, Nassau, Bahamas

The Bahamas is a group of islands, just off the coast of Florida, extending southwards to near the island of Hispaniola. There are approximately 25 inhabited islands and numerous small islands and cays that are uninhabited. The population of the Bahamas is approximately 230-240,000. A census taken in 1980 showed that approximately $65 \%$ of the population is aged between $15-45$ years. The major industries lie in tourism and off-shore banking. The Bahamas has not always had a drug problem; the diagnostic records at the mental hospital, Sandilands Rehabilitation Centre, reveal that in the sixties drug-related admissions were negligible. In the early seventies, however, there was a rapid increase in cannabis use and at the same time the number of drug-related admissions increased. The patients were mainly admitted in acutely disturbed states which were thought to be drug psychoses caused by cannabis inhalation. There do not seem to have been many patients requesting help to stop using cannabis, and generally there has been no significant change in this pattern. The major difference is the vast increase in the quantities smoked by an individual user. In the mid-seventies, the pattern of drug use began to change in that more patients were using a number of drugs at the same time. Methaqualone enjoyed a period of popularity and was known on the street as Quaaludes, Mx or Disco Biscuit. The drug's ability to potentiate the action of alcohol made it both dangerous and alluring, as it was possible to get 'stoned' almost immediately for the price of only one drink and one pill. It was about the same time in the mid-seventies that cocaine hydrochloride began to arrive on the market in increasing quantities and start gaining in popularity. The major problems at that time were associated with druginduced psychoses, acute intoxications and occasions of violent bizarre behaviour due to the disinhibition and release of aggression caused by these drugs. One drug addict from this era, interviewed in the prison, said that a good night out was to smoke some grass, drink a Guinness stout, take a quaalude and then snort some cocaine. There is really no literature as to what effect these combinations may have on the brain. It is of interest however that very few patients were presenting for treatment of addiction, though by the late seventies a trickle had begun to come forward. Cocaine was not that plentiful and was being used by snorting, i.e. the inhalation of the finely ground white powder into the nostrils. A less popular method of use of cocaine at that time was smoking the finely ground powder as a constituent of a tobacco cigarette or mixed into the contents of a marijuana cigarette. The quantities of cocaine available on the Bahamian street market at that time were small.

The next major change in drug abuse patterns occurred around 1979 when somehow the concept of 'freebasing' cocaine was introduced to the Bahamas. The problem was not immediately apparent, cases began to surface and stories indicating the depths of this addiction began to worry us. The admission rate gradually began to increase, but cocaine was still expensive and so many people could not afford it.

Over the last three years the method of use known as free-basing has become increasingly popular, so much so that $98 \%$ of all patients seeking help with a cocaine dependence problem report free-basing as their method of use. The method involves releasing relatively pure cocaine from the hydrochloride radical, by a simple process involving the addition of an alkali such as sodium bicarbonate, dissolving both in water and heating the mixture. After cooling pure cocaine crystals are formed. The pure cocaine is then vapourised and inhaled using a variety of instruments. The most commonly used is a home-made pipe, locally referred to as a 'camoke', made by covering a glass with tinfoil, making small holes in one side, on which cigarette ash and the cocaine crystals are placed, and placing another larger hole in the opposite side, which serves as a mouthpiece. Patients report that the cocaine is now more often than not sold to them already prepared for 'free-basing'. In 1982 most of the patients admitted to psychiatric hospital for help with a cocaine dependence problem were males between the ages of 18 and 30 years and, although all social classes were represented, more than $50 \%$ of these patients were from the lower socio-economic groups.

Over 1983 and the first half of 1984 there have been a number of changes noted. Firstly, the number of patients seeking help for cocaine dependence has increased to such 